\title{
Test-Retest Reliability of Single-Leg Time to Stabilization Following a Drop-Landing Task in Healthy Individuals
}

\author{
Aaron Byrne, Clare Lodge, and Jennifer Wallace
}

\begin{abstract}
Context: Single-leg stability has been associated with injury risk and is a key component of many injury prevention interventions. Methods of measuring single-leg stability are varied yet often unreliable. Objective: To establish within- and between-day testretest reliability for single-leg time to stabilization (SL-TTS) following a drop-landing maneuver of $20 \mathrm{~cm}$ in height among a healthy cohort. Design: Test-retest reliability study. Setting: Healthy cohort from a third-level educational institution. Participants: Nineteen (11 females and 8 males) healthy individuals. Main Outcome Measures: The SL-TTS in the vertical plane. Results: The SL-TTS showed good within-day (intraclass correlation coefficient $=.715$ ) and excellent between-day (intraclass correlation coefficient $=.83$ ) test-retest reliability. The minimal detectable change was calculated as $171.6 \mathrm{~ms}$ for within-day contexts and $123.8 \mathrm{~ms}$ for between-day contexts. Conclusions: This method of measuring SL-TTS is reliable and could be used to detect changes over time in a healthy cohort. This could be of value to clinicians in injury risk factor identification or assessing the effectiveness of single-leg stability training. However, further research is needed to investigate its reliability in pathological populations.
\end{abstract}

Keywords: force plate, outcome measure, protocol, dynamic stability

Deficits in landing stability have been identified as a risk factor for the incidence of ankle sprains and knee ligament sprains, ${ }^{1}$ along with a plethora of other lower-limb injuries. ${ }^{2}$ Landing stability has been a focus of many injury prevention programs, such as the FIFA 11 and FIFA $11+,{ }^{3}$ which are recognized worldwide. Measuring landing stability has been aided by technological advancements and increased accessibility to force platforms, meaning clinicians can now quantify ground reaction forces (GRFs) and their translation across multiple planes during landing tasks. This information may be of value to rehabilitation clinicians, as previous research has identified that deficits in landing stability are a risk factor for lower-extremity injuries. ${ }^{1}$ Traditionally, motion capture and 3-dimensional kinematic analyses have been utilized to measure landing stability; however, these methods are often more expensive, time consuming, and financially exhaustive when compared with force plate-derived outcome measures.

Time to stabilization (TTS) is a frequently reported metric which measures the time taken to stabilize to within a preset stability threshold after landing. ${ }^{4}$ However, TTS has been shown to be an unreliable outcome, with its variability attributed to factors such as lack of consistency in the propulsive jump prior to landing, lack of control for arm swing during jumping tasks used, or the complex demands placed on participants through reaching for the Vertec vertical jump apparatus (Gill Athletics, Champaign, IL), before placing hands back on the hips prior to landing. ${ }^{5}$

The aim of this study was to design a new protocol to reliably assess single-leg TTS (SL-TTS), while addressing the previously highlighted limitations in existing procedures (with the use of a protocol which attempts to control many of the aforementioned limitations). This proposed protocol measured single-leg TTS,

The authors are with the Department of Science and Health, Institute of Technology Carlow, Carlow, Ireland. Byrne (Aaron.Byrne@itcarlow.ie) is corresponding author. following a drop from a fixed $20 \mathrm{~cm}$ height, in a healthy cohort using a custom excel calculation spreadsheet.

\section{Methods}

\section{Participants}

A sample of 19 (11 females and 8 males) physically active thirdlevel students (aged 22.4 [2.2] y) were voluntarily recruited through convenience sampling from the Department of Science and Health and Sports Department of the Institute of Technology Carlow (Table 1). Subjects were deemed eligible if they participated, for more than 2 hours per week, in a field sport. Subjects were excluded if they reported any incidence of back or lower-limb injury in the past 6 months. An injury was defined as any injury which prevented them from participating at full capacity in their chosen sport for more than 24 hours after the injury occurred. ${ }^{6}$ Participants were informed of the potential risks and benefits associated with their participation in the study. Informed consent and medical screening forms were obtained, as approved by the ethics in research committee of the Institute of Technology Carlow, Ireland. Subjects were informed that they were free to withdraw from the study at any time. The single tester, a Certified Athletic Therapist, with 3 years of experience, was blinded to all outcomes as all data were retrospectively analyzed after study completion.

\section{Procedures}

All measurements were performed by a single tester, a Certified Athletic Therapist, over 2 testing sessions, 3 days apart, in the Physiology Laboratory at Institute of Technology Carlow. Both testing sessions occurred at the same time on each of the days with subjects requested to abstain from high-intensity exercise for 24 hours prior to testing. All measurements were performed on the participants' self-reported preferred jumping leg (leg which they would prefer to jump off when jumping for a ball). 
The SL-TTS was recorded using the HUR labs Force Platform 8 (FP8; HUR labs Oy, Tampere, FI) in "General Acquisition of Data" mode, with all 4 sensors recording vertical GRFs at a refresh rate of $1000 \mathrm{~Hz}$, taking sum of all sensor values as the final output measure. Individual body mass was measured in newtons by the force platform. Participants stood barefoot on the $28-\mathrm{cm}$ high box with toes at the edge. Subjects kept their hands on their hips at all times and were instructed to "step forward from a straight back leg, land and stick that landing position for a total of seven seconds" as counted aloud by the tester. As the FP8 stood at $8 \mathrm{~cm}$ high, the total drop height was $20 \mathrm{~cm}$ from box to the landing zone, which was $81 \times 61 \mathrm{~cm}$ in dimension. Trials were repeated if they removed their hands, added a hop in order to stabilize, or put their second foot down before the 7 seconds had elapsed (Figure 1).
Five trials were performed as familiarization on each testing day to allow subjects to become comfortable with the test. Four recorded trials were then performed with raw data retrospectively exported for posttesting analysis. A 30-second rest period was adhered to during all familiarization and recorded trials.

The raw data were then inserted into a custom-created excel spreadsheet containing formulae to calculate the time at which stability was reached. Stability was defined as the point at which vertical GRF reached a level within $5 \%$ of body weight and remained within $5 \%$ of body weight for a subsequent second (1000 data points).$^{5,7}$ The time point which corresponded with the first point at which vertical GRF crossed $10 \mathrm{~N}$ was accepted as initial contact time and was subtracted from the onset of stability to calculate SL-TTS (see Supplementary Material [available online] for spreadsheet). The 3 best times (quickest) out of the

Table 1 Mean and SD for Subject Age, Body Mass, and Self-Selected Preferred Jumping Leg

\begin{tabular}{|c|c|c|c|c|c|}
\hline Characteristic & Female $(n=11)$ & \multicolumn{2}{|c|}{ Male $(n=8)$} & \multicolumn{2}{|c|}{ Total $(\mathrm{N}=19)$} \\
\hline Age, $y$ & $22.1(2.7)$ & \multicolumn{2}{|c|}{$22.8(1.4)$} & \multicolumn{2}{|c|}{$22.4(2.2)$} \\
\hline Body mass, $\mathrm{kg}$ & $67.7(10.6)$ & \multicolumn{2}{|c|}{$83(15)$} & \multicolumn{2}{|c|}{$74.1(14.5)$} \\
\hline Preferred jumping leg & $\mathrm{L}=7$ & $\mathrm{~L}=5$ & $\mathrm{R}=3$ & $\mathrm{~L}=12$ & $\mathrm{R}=7$ \\
\hline
\end{tabular}

Abbreviations: L, left; R, right.

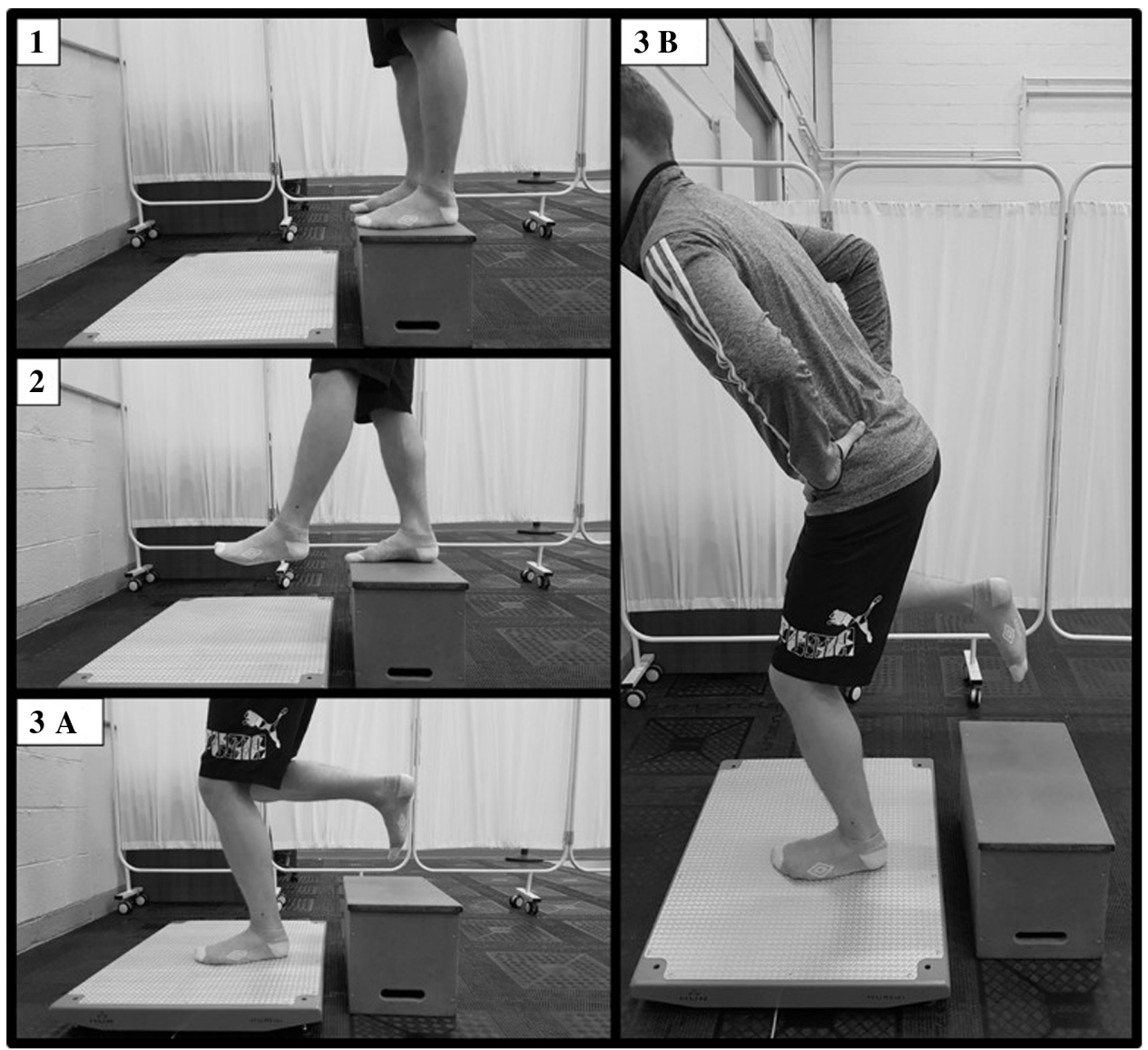

Figure 1 - Single-leg time to stabilization procedures. 
4 test trials were used for analysis upon completion of testing procedures.

\section{Statistical Analyses}

All data were tabulated and analyzed using IBM Statistical Package for the Social Sciences (SPSS, version 23, Armonk, NY) and Microsoft Excel 2013 (Redmond, WA). Means, SDs, coefficients of variance in percentage, and intraclass correlation coefficients (ICCs), along with the respective $95 \%$ confidence intervals were calculated within SPSS with $\alpha=.05$ and $1-\beta=0.95$. ICC $(3,1)$ was applied in within-day analyses, with ICC $(3, k)$ applied in betweenday analyses for intrarater reliability ${ }^{8-10}$ The ICC statistics were classified within the following ranges: poor (.0-.39), fair (.4-.59), good (.6-.74), or excellent (.75-1). ${ }^{8}$ The SEM and minimal detectable change (MDC ${ }^{95}$ ) were calculated for both within- and betweenday reliability analyses using the following formulae. ${ }^{8,9}$

$$
\mathrm{SEM}=\mathrm{SD} \times \sqrt{ }(1-r)
$$

(with " $r$ " being the ICC value calculated prior),

$$
\mathrm{MDC}^{95}=1.96 \times \sqrt{ }(2) \times \mathrm{SEM} .
$$

\section{Results}

Mean and SD SL-TTS scores for the 3 best trials recorded on day 1 and mean and SD scores for days 1 and 2 are represented in Table 2. Good within-day reliability $(\mathrm{ICC}=.715)$ was found across the trials for day 1 with ICC $(3,1), 95 \%$ confidence intervals, coefficients of variance, SEM, and $\mathrm{MDC}^{95}$ represented in Table 2. On comparison of days 1 and 2 average measures, excellent between-day reliability $(\mathrm{ICC}=.83$ ) was found with ICC $(3, k), 95 \%$ confidence intervals, coefficients of variance, SEM, and $\mathrm{MDC}^{95}$ represented in Table 2. All participants successfully completed testing procedures with the maximum number of failed trials which required repetition being 1 .

\section{Discussion}

The aim of this study was to establish the reliability of SL-TTS measurement after a standardized drop-landing task. Findings suggest that there is good within-day intratester reliability (ICC $[3,1]=.715$ ) and excellent between-day intratester reliability (ICC
$[3, k]=.83$ ) for the SL-TTS, which exceeds those reported previously (ICCs $=.423$ and .662 , respectively). ${ }^{5}$ Furthermore, the reported $\mathrm{MDC}^{95}$ values provide valuable information to practitioners that any change in SL-TTS greater than $124 \mathrm{~ms}$ means a true change in performance has occurred beyond that of measurement error alone. ${ }^{9}$

This degree of reliability may have been achieved by removing arm movement and visual demands which were present in previous procedures, ${ }^{5}$ while also replacing the propulsive jump with a step-down from a standardized height of $20 \mathrm{~cm}$, although this may be a study limitation as the landing task may not reflect a situation which occurs in sporting contexts. ${ }^{5}$ Finally, through the use of a custom excel spreadsheet for SL-TTS calculations, this outcome can be cheaply and easily measured by any practitioner once their chosen force plate can measure and plot vertical GRF with its corresponding time data, as opposed to employing the labor of software developers to design a similar testing protocol.

This procedure allows clinicians to measure one's ability to stabilize following a single-leg landing in a reliable and economical manner.

\section{Study Limitations}

Although reliable, the adapted testing procedures may not replicate sporting demands as landings rarely occur in a controlled manner, like those in the outlined experimental procedure. Also, investigation into the task-specific learning effects and reliability of these procedures in pathological populations is necessary before it is widely accepted into clinical practice.

Future research should aim to investigate the intertester reliability and external validity of SL-TTS measurement using this protocol, comparison to a gold standard such as 3-dimensional kinematic data, its association with injury incidence, and its reliability among pathological populations and specific target populations (athletes, etc) to allow greater generalization of outcomes.

\section{Acknowledgments}

The authors would like to thank the Department of Science and Health in Institute of Technology Carlow and the students who volunteered their time to participate in this study. The authors declare no conflicts of interest with regard to research, authorship, formatting, or dissemination. No external funding was received for this current research.

\begin{tabular}{|c|c|c|c|c|c|c|c|}
\hline \multirow[b]{2}{*}{ Movement } & \multirow[b]{2}{*}{ Trial $1, \bar{x}(S D)$} & \multicolumn{6}{|c|}{ Within-day reliability statistics for SL-TTS } \\
\hline & & Trial $2, \bar{x}(\mathrm{SD})$ & Trial $3, \bar{x}(\mathrm{SD})$ & $\operatorname{ICC}(3,1)(95 \% \mathrm{Cl})$ & CV\% & SEM & MDC $^{95}$ \\
\hline \multirow[t]{2}{*}{ SL-TTS } & $749.1(296.7) \mathrm{ms}$ & $698.7(283.6) \mathrm{ms}$ & $671.3(234.2) \mathrm{ms}$ & $.715(.498-.867)$ & $14.7 \%$ & $61.9 \mathrm{~ms}$ & $171.6 \mathrm{~ms}$ \\
\hline & \multicolumn{7}{|c|}{ Between-day reliability statistics for SL-TTS } \\
\hline Movement & Day $1, \bar{x}(\mathrm{SD})$ & \multicolumn{2}{|c|}{ Day $2, \bar{x}(\mathrm{SD})$} & ICC $(3, k)(95 \% \mathrm{Cl})$ & CV\% & SEM & MDC $^{95}$ \\
\hline SL-TTS & $706.4(245.6) \mathrm{ms}$ & $616.4(230.8) \mathrm{ms}$ & \multicolumn{2}{|c|}{$.830(.559-.935)$} & $16.1 \%$ & $44.7 \mathrm{~ms}$ & $123.8 \mathrm{~ms}$ \\
\hline
\end{tabular}

Table 2 Within- and Between-Day Reliability Statistics

Abbreviations: $\bar{x}$, mean; CI, confidence interval; $\mathrm{CV}$, coefficient of variance, expressed as a percentage; ICC (3,1), intraclass correlation coefficient-2-way mixed-effects, single measures; ICC $(3, k)$, intraclass correlation coefficient-2-way mixed-effects, average measures; MDC ${ }^{95}$, minimal detectable change at $95 \%$ CI; SL-TTS, single-leg time to stabilization. 


\section{References}

1. De Blaiser C, Roosen P, Willems T, Danneels L, Bossche LV, De Ridder R. Is core stability a risk factor for lower extremity injuries in an athletic population? A systematic review. Phys Ther Sport. 2018;30:4856. PubMed ID: 29246794 doi:10.1016/j.ptsp.2017.08.076

2. Murphy DF, Connolly DAJ, Beynnon BD. Risk factors for lower extremity injury: a review of the literature. Br J Sports Med. 2003; 37(1):13-29. PubMed ID: 12547739 doi:10.1136/bjsm.37.1.13

3. Thorborg K, Krommes KK, Esteve E, Clausen MB, Bartels EM, Rathleff MS. Effect of specific exercise-based football injury prevention programmes on the overall injury rate in football: a systematic review and meta-analysis of the FIFA 11 and $11+$ programmes. $\mathrm{Br} \mathrm{J}$ Sports Med. 2017;51(7):562-571. PubMed ID: 28087568 doi:10. 1136/bjsports-2016-097066

4. Fransz DP, Huurnink A, de Boode VA, Kingma I, van Dieen JH. Time to stabilization in single leg drop jump landings: an examination of calculation methods and assessment of differences in sample rate, filter settings and trial length on outcome values. Gait Posture. 2015;41(1): 63-69. PubMed ID: 25242295 doi:10.1016/j.gaitpost.2014.08.018
5. Flanagan EP, Ebben WP, Jensen RL. Reliability of the reactive strength index and time to stabilization during depth jumps. J Strength Cond Res. 2008;22(5):1677-1682. PubMed ID: 18714215 doi:10. 1519/JSC.0b013e318182034b

6. Brooks JHM, Fuller CW, Kemp SPT, Reddin DB. Epidemiology of injuries in English professional rugby union: part 1 match injuries. $\mathrm{Br}$ J Sports Med. 2005;39(10):757-766. PubMed ID: 16183774 doi:10. 1136/bjsm.2005.018135

7. Wikstrom EA, Powers ME, Tillman MD. Dynamic stabilization time after isokinetic and functional fatigue. J Athl Train. 2004;39(3):247253. PubMed ID: 15496994

8. Weir JP. Quantifying test-retest reliability using the intraclass correlation coefficient and the SEM. J Strength Cond Res. 2005;19(1): 231-240. PubMed ID: 15705040 doi:10.1519/15184.1

9. Portney LG, Watkins MP. Foundations of Clinical Research: Applications to Practice. New York, NY: Pearson Education; 2009:892.

10. Koo TK, Li MY. A guideline of selecting and reporting intraclass correlation coefficients for reliability research. J Chiropr Med. 2016; 15(2):155-163. PubMed ID: 27330520 doi:10.1016/j.jcm.2016. 02.012 